\title{
Ubiquitous Body Sensor Networks
}

\author{
Guest Editors Ubiquitous Body Sensor Networks • \\ Min Chen • Athanasios V. Vasilakos
}

Published online: 13 August 2011

(C) Springer Science+Business Media, LLC 2011

Recently, there has been a lot of increasing investigation on a new type of wireless sensor network, which is generally known as body sensor networks (BSNs). Following the continuous advances in wireless communications technologies, there is a growing interest in the design and development of BSNs systems for applications of improving people's daily life, which leads to an increasing demand on interconnecting BSNs with other emerging wireless technologies, such as RFID technology, wireless sensor networks (WSNs), Zigbee, Bluetooth, WiBree, video surveillance system, WPAN, WLAN, internet, and cellular network. With BSNs being incorporated into these technologies, BSNs become ubiquitous. The marketing opportunities for advanced consumer electronics and services will be expanded extensively. More and more autonomous and intelligent applications which are close to human's life will be generated. For example, by using body sensors to collect patient information, and by deploying diverse wireless networking systems for enhanced diagnosis assistance and action handling, better E-healthcare management system can be designed for providing more desirable services, which implies huge market yet to be discovered, due to an increasing demand of E-healthcare management system in recent years.

This special issue is intended to provide a forum for presenting, exchanging and discussing the recent advances

\footnotetext{
M. Chen $(\bowtie)$

Seoul National University,

Seoul, Korea

e-mail: minchen@ece.ubc.ca

A. V. Vasilakos

National Technical University of Athens,

Athens, Greece

e-mail: vasilako@ath.forthnet.gr
}

on body sensor networks in terms of communications and networking, security and privacy, and systems and technology. In response to the call for contributions, we have received a relatively large number of papers from both academia and industries that covered a variety of interesting topics related to BSNs. Two rounds of careful review by the guest editors and experts in the field led to five papers for inclusion in this half issue.

The first paper "Transmission of Patient Vital Signs using Wireless Body Area Networks" by B. Chen and D. Pompili proposed an interference-aware networking solution for healthcare monitoring during in-hospital triage, where accurate and quick prioritization of patient vital signs is crucial for making efficient and real-time emergency medical care decisions. It aims to provide an electromagnetic-interference-aware communication solution for in-hospital networks with light and moderate congestion with the objective to maximize the reliability of vital signs while guaranteeing their end-to-end delay requirements. A cross-layer communication solution jointly considering three functionalities - routing, medium access control, and scheduling - was designed to support the interference-aware prioritization services. By providing better wireless channels to patients who need more immediate services and scheduling packets based on the patient's condition and data requirements, the proposed solution offers higher reliability and lower delay for patients who need immediate medical treatment. Field experiments show that the proposed solution is effective in achieving the design objectives. And it can offer better performance than conventional wireless protocols.

In the paper "A Secure Handshake Scheme with Symptoms-Matching for mHealthcare Social Network" by $\mathrm{R}$. $\mathrm{Lu}$ et al., the authors addressed security and privacy issues in mHealthcare social network (MHSN) - an appli- 
cation of ubiquitous body sensor network. MHSN can provide a promising platform for the seniors who have the same symptom to exchange their experiences, give mutual support and inspiration to each other, and help forwarding their health information wirelessly to a related eHealth center. However, there exist many challenging security and privacy issues in MHSN such as how to securely identify a senior who has the same symptom, how to prevent others who don't have the symptom from knowing someone's symptom? In this work, the authors propose a secure handshake scheme with symptoms-matching to tackle these challenging issues. The other paper addressing privacy issue is entitled by "Temporal Accountability and Anonymity in Medical Sensor Networks". In this work, J. Liu and Y. Xiao proposed a new architecture for the medical sensor network that is capable of temporal accountability, while preserving privacy ability. The analysis results clearly indicate the advantages of being proposed methods in terms of low-cost and reliable and having scalable features.

O. Pereira, J. Caldeira and J. Rodrigues, in the paper "A Mobile Core-Body Temperature Monitoring System on Android", presented four mobile solutions for continuous biofeedback monitoring of a BSN. It includes Symbian, Android, Windows Mobile, and iPhone operating systems.
All the proposed mobile applications can collect, visualize, and monitoring data from BSN sensors in both real-time and off-line modes. The validation of the proposed systems was performed through different equipments with success and it was proven they are ready for use.

In the last paper "Mobile Device Aided Cooperative Transmission for Body Area Networks", R. Yu et al. studied the potential of cognition and cooperation in Body Area Networks (BANs), and proposed a network architecture for cognitive and cooperative communications in BANs. An intelligent mobile device is introduced as either a cognitive gateway to interconnect heterogeneous networks; or a cooperative relay node to achieve transmission diversity. Two cooperative transmission schemes, Energy-conserved Cooperative Transmission and Reliability-driven Cooperative Transmission, are presented for different applications that have distinct energy consumption or reliability requirement.

We would like to thank all the reviewers for their efforts and constructive comments. We would in particular like to thank Professor Imrich Chlamtac, the Editor-in-Chief, for his support and helpful suggestions during the very delicate stages of concluding the special issue. Finally, we would like to thank all the authors who submitted their precious research work to this special issue. 